\title{
Featureless EMG pattern recognition based on convolutional neural network
}

\author{
Jingwei Too ${ }^{1}$, A. R. Abdullah ${ }^{2}$, N Mohd Saad ${ }^{3}$, N Mohd Ali $^{4}$, T. N. S. Tengku Zawawi ${ }^{5}$ \\ ${ }^{1,2,4,5}$ Fakulti Kejuruteraan Elektrik, Universiti Teknikal Malaysia Melaka, Malaysia \\ ${ }^{3}$ Fakulti Kejuruteraan Elektronik dan Kejuruteraan Komputer, Universiti Teknikal Malaysia Melaka, Malaysia
}

\begin{tabular}{l} 
Article Info \\
\hline Article history: \\
Received Jun 10, 2018 \\
Revised Nov 18, 2018 \\
Accepted Jan 21, 2019 \\
\hline Keywords: \\
Convolutional neural network \\
Electromyography \\
Pattern recognition \\
Spectrogram
\end{tabular}

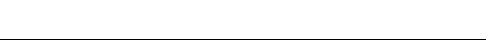

\section{Corresponding Author:}

A. R. Abdullah,

Fakulti Kejuruteraan Elektrik,

Universiti Teknikal Malaysia Melaka,

Jalan Hang Tuah Jaya, 76100 Durian Tunggal, Melaka, Malaysia.

Email: abdulr@utem.edu.my

\begin{abstract}
Feature extraction is important step to extract the useful and valuable information from the electromyography (EMG) signal. However, the process of feature extraction requires prior knowledge and expertise. In this paper, a featureless EMG pattern recognition technique is proposed to tackle the feature extraction problem. Initially, spectrogram is employed to transform the raw EMG signal into time-frequency representation (TFR). The TFRs or spectrogram images are then directly fed into the convolutional neural network $(\mathrm{CNN})$ for classification. Two $\mathrm{CNN}$ models are proposed to learn the features automatically from the spectrogram images without the need of manual feature extraction. The proposed CNN models are evaluated using the EMG data acquired from the publicly access NinaPro database. Our results show that $\mathrm{CNN}$ classifier can offer the best mean classification accuracy of $88.04 \%$ for the recognition of the hand and wrist movements.
\end{abstract}

Copyright $\odot 2019$ Institute of Advanced Engineering and Science. All rights reserved.

\section{INTRODUCTION}

In recent days, electromyography (EMG) has shown its potential in biomedical, clinical, myoelectric prosthetic and rehabilitation applications. Generally, EMG signal is one of the biological signals that measures the potential difference generated by the muscle fibre during muscle contraction [1]. Additionally, EMG signal provides rich muscle and motor information, which allows the myoelectric prosthetic control for nearly 40 years [2]. In this regard, many researchers have paying high attentions in analyzing the EMG signal for controlling the electrical powered prosthetic [3]. An EMG based prosthetic hand helps the amputee and stroke patients to restore their limbs functionality [2], [4]. Despite the advance in machine learning, the development of multifunctional myoelectric prosthetic has been increased. However, an accurate multifunction prosthetic is still a challenging task in the real time applications [4], [5].

Machine learning has recently drawn the attention of researchers in EMG pattern recognition. By applying machine learning algorithm, the performance of myoelectric prosthetic has shown great improvement [6]. Nevertheless, feature extraction is a critical step for achieving a good recognition rate in myoelectric control. In the previous studies, time domain (TD), frequency domain (FD) and time-frequency (TF) features were widely used in EMG pattern recognition [7]-[10]. However, the selection and the number of features are mostly empirical and require expertise. Furthermore, the performances of features are often inconsistent, which leads to unsastisfactory classification results [11]-[13]. Hence, the deep convolutional neural network $(\mathrm{CNN})$ is introduced to eliminate the need of manual feature extraction in this work.

CNN is one of the popular deep learning methods that has been successfully applied in the classification of high dimensional data especially for image [14], [15]. In signal processing, the signal can be 
transformed into time-frequency representation (TFR) using the time-frequency distribution (TFD). The TFD images, which can be used as the input on CNN for diagnosis, classification and identification. In one study, Wang et al. [12] diagnosed the fault detection by using a series of wavelet scalogram as an input for CNN without manual feature extraction. Furthermore, Verstraete et al. [16] made use of spectrogram, HilbertHuang Transform (HHT) and wavelet scalogram as the input in CNN for fault detection. From the previous works, it shows that TFD have been successfully implemented in CNN model for classification tasks.

This study aims to investigate the performance of CNN model when classifying the basic hand and wrist movements. Firstly, the EMG data of 11 amputees and 10 healthy subjects are acquired from NinaPro databases. After that, spectrogram is applied to transform the EMG signals into TFRs or spectrogram images, and then fed into the CNN for recognition process. In this work, two CNN architectures have been proposed and tested on both healthy and amputee datasets. At last, the performances of proposed CNN models are discussed and validated with statistical analysis. In short, this article describes the performance of featureless EMG pattern recognition for the classification of multiple hand movement types.

\section{MATERIAL AND METHOD}

\subsection{Materials}

In this work, the EMG data from the Non-Invasive Adaptive Prosthetics (NinaPro) project is used. Recently, NinaPro database has been successfully applied in EMG pattern recognition studies [17], [18]. In the present work, the EMG data of 17 hand and wrist movements (Exercise B) including thumb up (M1), extension of index, middle and flexion of others (M2), flexion of ring, little and extension of others (M3), thumb opposing base of little finger (M4), abduction of all fingers (M5), fingers flexed together in fist (M6), pointing index (M7), adduction of extended fingers (M8), wrist supination with axis at middle finger (M9), wrist pronation with axis at middle finger (M10), wrist supination with axis at little finger (M11), wrist pronation with axis at little finger (M12), wrist flexion (M13), wrist extension (M14), wrist radial deviation (M15), wrist ulnar deviation (M16) and wrist extension with closed hand (M17) are utilized [5]. The EMG signals are collected from NinaPro database 3 (DB3) and database 4 (DB4), which comprises of 11 amputees and 10 healthy subjects, respectively [19], [5]. The EMG signal was sampled at $2 \mathrm{kHz}$, however in this work the signal was initially sub-sampled by a decimation factor of 2 . In the experiment, each movement was performed for 5 seconds and followed by a resting state of 3 seconds. Subjects were instructed to repeat each movement types for six times. In total, 1224 EMG signals $(17$ hand movement types $\times 6$ repetitions $\times$ 12 channels) were collected from each subject. Moreover, the generalized likehood ratio algorithm was used in offline relabeling. Note that all the resting states are removed for DB3 and DB4.

\subsection{System Overview}

Figure 1 demonstrates the flow diagram of the proposed featureless EMG pattern recognition system. In the first step, the EMG signals are collected from DB3 and DB4. The sample EMG signal obtained from one subject was shown in Figure 2. After that, spectrogram is applied to transform the signal into TFR. Next, the TFRs (spectrogram images) are directly fed into the CNN for predicting the 17 hand and wrist movements.

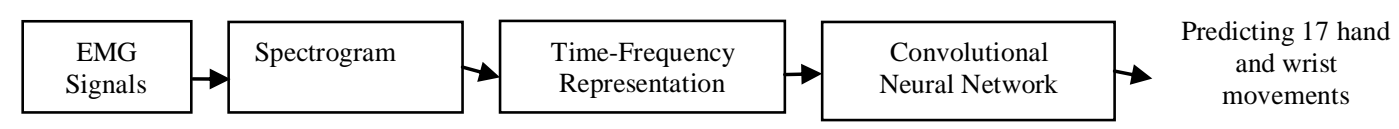

Figure 1. Flow diagram of proposed featureless EMG pattern recognition system

\subsection{Spectrogram}

Spectrogram is the square magnitude of short time Fourier transform (STFT), and it is defined as the visual representation of the STFT in time-frequency plane [16]. In spectrogram, the X-axis represents the time domain while the y-axis is referred to the frequency domain. Spectrogram provides the relationship between the EMG signal and muscle characteristic, which describes the muscle behavior for different hand movements [20]. Mathematically, spectrogram can be represented as:

$$
S(t, f)=\left|\int_{-\infty}^{\infty} x(\tau) w(\tau-t) e^{-2 \pi f \tau} d \tau\right|^{2}
$$


where $\mathrm{x}(\tau)$ is the input signal and $\mathrm{w}(\tau-\mathrm{t})$ is the Hanning window function. In this work, spectrogram with 128 ms (128 samples) and 50\% overlap (64 samples) is used. Additionally, spectrogram is computed using 128 Fourier (FFT) points.

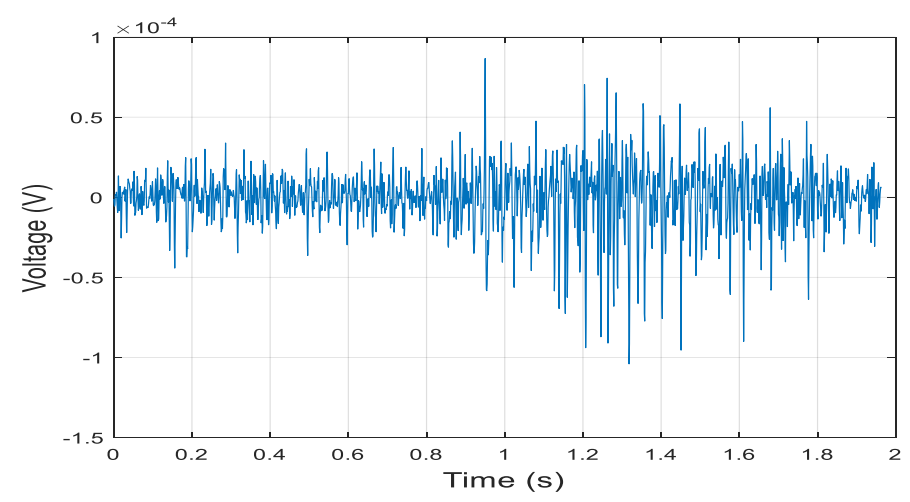

Figure 2. Sample EMG signal obtained from one subject

In this framework, spectrogram generated from twelve different channels are combined into a series image. After that, the spectrogram images are resized to $64 \times 64$ pixel and fed into the CNN for the classification process. Figure 3 illustrates the sample spectrogram images for 17 hand and wrist movements obtained from one subject. The color scale represents the amplitude of signal energy. From Figure 3, one can see that different type of hand movement offered different kind of visual representation. Especially M13, which provides a significant difference in spectrogram image as compared to other movements.

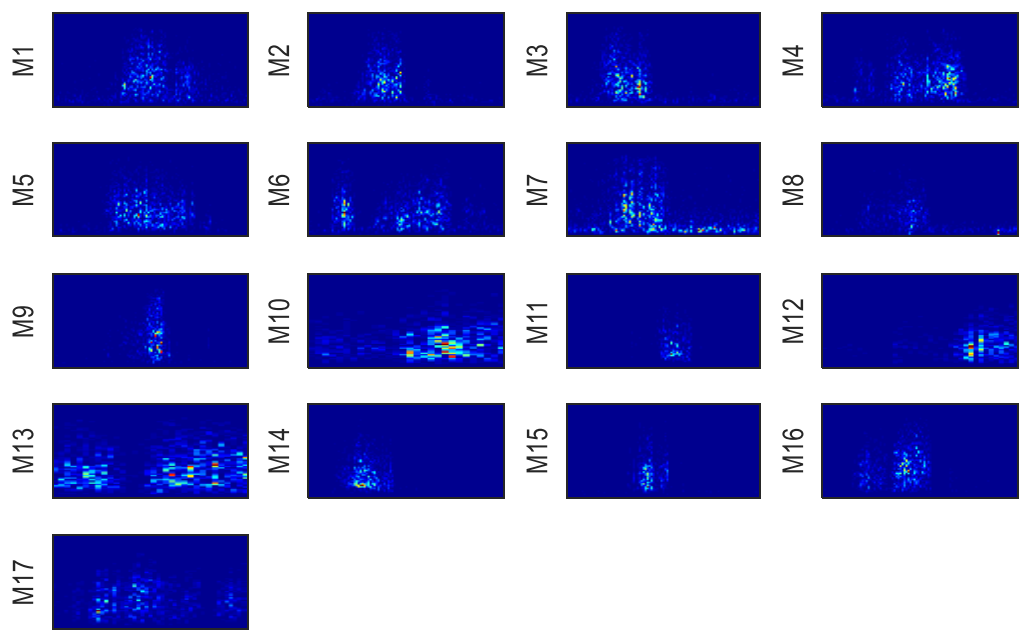

Figure 3. Sample spectrogram images for 17 hand and wrist movements

\subsection{Convolutional Neural Network}

Convolutional neural network (CNN) is one of the best supervised deep learning algorithm for the classification of high dimensional images [14]. Generally, CNN takes the spectrogram images as the input directly for the classification process, thus avoiding the high complexity of pre-processing and feature extraction [12]. Instead of manually feature extraction, CNN offers the automatic feature extraction using the concept of deep neural network. Basically, CNN comprises of three main layers namely convolutional layer, max pooling layer and fully connected layer. At the first stage, convolutional layer fixes up the units in a sequence of filters. The width and height of input image in each filter are convolved during the training process [21], [22]. For the second stage, the max pooling layer produces a non-linear sub-sampling. This subsampling aims to reduce the dimension of input, thus reducing the amount of features in the training network 
[23]. In the final stage, the fully connected layer multiples the input by weight matrix and carries out the classification process [24], [25]. In short, convolutional and max pooling layers construct the features from the images while the classification process is executed by the fully connected layer.

\subsection{Proposed Model Architecture}

In this work, two CNN models are proposed for featureless EMG pattern recognition. Table 1 and 2 outline the overview of CNN architecture 1 and 2, respectively. In Table 1, it shows that CNN architecture 1 consisted of 5 layers. The first stage consisted of a convolutional layer of 32 feature maps of $3 \times 3$ size. It is followed by a max pooling layer of $2 \times 2$ size with stride of 2 . The final stage including a fully connected layer and a Softmax layer.

From Table 2, it is observed that CNN architecture 2 had an additional convolutional and max polling layers compared to $\mathrm{CNN}$ architecture 1. As can be seen, $\mathrm{CNN}$ architecture 2 made use of the convolutional layers with 16 and 32 filters for first and second convolutional layers, respectively.

Table 1. Overview of CNN Architecture 1

\begin{tabular}{ccccc}
\hline Layer & Layer Type & Filter Number & Filter Size & Stride Size \\
\hline 1 & Convolutional & 32 & $3 \times 3$ & - \\
2 & Max Pooling & 1 & $2 \times 2$ & 2 \\
3 & Fully Connected & 17 & $64 \times 64$ & - \\
\hline
\end{tabular}

Table 2. Overview of CNN Architecture 2

\begin{tabular}{|c|c|c|c|c|}
\hline Layer & Layer Type & Filter Number & Filter Size & Stride Size \\
\hline 1 & Convolutional & 16 & $3 \times 3$ & - \\
\hline 2 & Max Pooling & 1 & $2 \times 2$ & 2 \\
\hline 3 & Convolutional & 32 & $3 \times 3$ & - \\
\hline 4 & Max Pooling & 1 & $2 \times 2$ & 2 \\
\hline 5 & Fully Connected & 17 & $64 \times 64$ & - \\
\hline
\end{tabular}

For both CNN model 1 and 2, the convolutional layer is followed by a batch normalization layer and rectified linear units (ReLU) layer. Batch normalization layer is applied to normalize the input by calculating the mean and standard deviation for each mini batch. The input is then normalized so that it has zero mean and unit variance. The normalize activation can be expressed as:

$$
x_{i}=\frac{x_{i}-\mu_{A}}{\sqrt{\sigma_{A}^{2}+e}}
$$

where $\mu \mathrm{A}$ is the mean, $\sigma \mathrm{A} 2$ is the variance and $\mathrm{e}$ is referred to the property epsilon. On one side, rectified linear unit (ReLU) layer is defined as a non-linear function that acts as a half-wave rectifier for arranging the weighted sum [16]. The convolutional function can be written as:

$$
Y_{k}^{(m)}=\operatorname{ReLU}\left(W_{k}^{(m)} * Y_{k-1}+B_{k}^{(m)}\right)
$$

where Yk-1 is the input for convolutional channel, * denotes convolutional manipulator, $\mathrm{Wk}(\mathrm{m})$ is the kernel filter weight, $\mathrm{Bk}(\mathrm{m})$ is the bias weight and ReLU can be represented as:

$$
\operatorname{ReLU}(y)=\max (y, 0)
$$

\section{RESULTS AND ANALYSIS}

In this paper, the classification performances of two proposed CNN architectures are investigated. The performances of CNN on both healthy and amputee subjects are also discussed. Remarkably, the TFR images generated by the spectrogram are used as the input directly for the classification process. All the analysis is done in Matlab 9.3 using computer with processing Intel Core i5-3340 3.1 GHz with 8 GB Random Access Memory (RAM). In the training phase, a batch size of 20 is applied to reduce the loss of the 
cross entropy. The initial learning rate is set at 0.01 and the maximum epochs of training process is fixed at 50. In order to eliminate the saturation information, the spectrogram images are converted into grayscale images. For performance evaluation, 6-fold cross validation is implemented. The data is randomly divided into six equal subsets and each subset is used to test in sequence. On one side, the remaining five subsets are used as the training samples.

\subsection{Performance Evaluation}

In order to investigate the effect of the number of convolutional layer on CNN model, two CNN architectures have been proposed and studied. Table 3 exhibits the classification accuracy of CNN model 1 and 2 for both healthy and amputee subjects. It is observed that the performances of healthy subjects are better than amputee subjects. In essence, healthy subjects often have a better performance than amputee subjects. This is expected since amputees have the difficulty in performing the hand movements without the complete upper limb. What the amputees did in the experiment is that they performed the movements based on their imaginations.

Table 3. Classification Accuracy of CNN Model 1 and 2 for Both Healthy and Amputee Subjects

\begin{tabular}{|c|c|c|c|c|}
\hline \multirow{3}{*}{ Subject } & \multicolumn{4}{|c|}{ Classification Accuracy (\%) } \\
\hline & \multicolumn{2}{|c|}{ Healthy Subject } & \multicolumn{2}{|c|}{ Amputee Subject } \\
\hline & CNN Model 1 & CNN Model 2 & CNN Model 1 & CNN Model 2 \\
\hline 1 & 85.29 & 88.24 & 76.47 & 79.41 \\
\hline 2 & 89.22 & 83.33 & 72.55 & 68.63 \\
\hline 3 & 85.29 & 88.24 & 38.24 & 45.10 \\
\hline 4 & 89.22 & 89.22 & 63.73 & 71.57 \\
\hline 5 & 86.27 & 86.27 & 35.29 & 34.31 \\
\hline 6 & 91.18 & 93.14 & 67.65 & 68.63 \\
\hline 7 & 88.24 & 95.10 & 35.29 & 31.37 \\
\hline 8 & 85.29 & 91.18 & 69.61 & 76.47 \\
\hline 9 & 82.35 & 85.29 & 72.55 & 79.41 \\
\hline 10 & 79.41 & 80.39 & 30.39 & 35.29 \\
\hline 11 & - & - & 69.61 & 73.53 \\
\hline Mean & 86.18 & 88.04 & 57.40 & 60.34 \\
\hline STD & 3.50 & 4.45 & 18.28 & 19.50 \\
\hline
\end{tabular}

As can be seen in Table 3, by applying CNN model 1, majority of healthy subject achieved the accuracy of above $80 \%$ except subject 10 . On the contrary, all the healthy subjects obtained the accuracy of above $80 \%$ when CNN model 2 is employed. Inspecting the results, for both healthy and amputee datasets, CNN model 2 achieved the highest mean classification accuracy of $88.04 \%$ and $60.34 \%$, respectively. By employing CNN model 2, the results showed an increment of $1.86 \%$ and $2.94 \%$ in mean classification accuracy for both healthy and amputee datasets. It is worth noting that CNN model 2 has an additional convolutional and max pooling layers. These finding indicates an increment of convolutional layer is able to enhance the quality of classification. This may be due to an additional process for automatic feature extraction in the CNN model. Based on the result obtained, it is clear that the selection of convolutional layer was equally important in designing a CNN architecture. Furthermore, the result of T-test shows that the classification performances of $\mathrm{CNN}$ model 1 and 2 are similar $(\mathrm{p}=0.1299)$ for healthy datasets. For amputee dataset, there is a significant difference $(\mathrm{p}=0.0484)$ between the classification performance of $\mathrm{CNN}$ model 1 and 2.

Table 4 summarizes the computational cost of CNN model 1 and 2 for both healthy and amputee subjects. Note that the computational time in Table 4 is the averaged results across 11 amputees and 10 healthy subjects. From Table 4, CNN model 2 spent nearly 6 minutes for the training and testing sessions. As compared to $\mathrm{CNN}$ model $1, \mathrm{CNN}$ model 2 took extra one minute to complete the classification task. Obviously, CNN model 1 was less time consuming as compared to model 2. This means that an additional layer in $\mathrm{CNN}$ model increased the computational complexity. Considering the classification accuracy and computational cost, it is believed that $\mathrm{CNN}$ model 2 is more appropriate to be applied in featureless EMG pattern recognition.

Table 4. Computational Cost of CNN Model 1 and 2

\begin{tabular}{ccc}
\hline \multirow{2}{*}{ Subject } & \multicolumn{2}{c}{ Average Computational time (s) } \\
& CNN Model 1 & CNN Model 2 \\
\hline Healthy & 295.09 & 337.42 \\
Amputee & 294.57 & 361.75 \\
\hline
\end{tabular}


In the final part of the evaluation, the mean class-wise accuracy of $\mathrm{CNN}$ model 2 for both healthy and amputee datasets are calculated as shown in Figure 4 and 5. From Figure 4, M1, M6, M9, M13, M14, M15, M16 and M17 are the most correct predicted (above 90\%) while the worst confusion rate is falling on M8 (76.3\%). From Figure 5, it is clear that the 17 hand and wrist movements performed by amputee subjects are difficult to recognize, especially M3 (51.5\%). As can be seen, the best recognition rate is found to be M1 (75.4\%), followed by M6, (71.4\%).

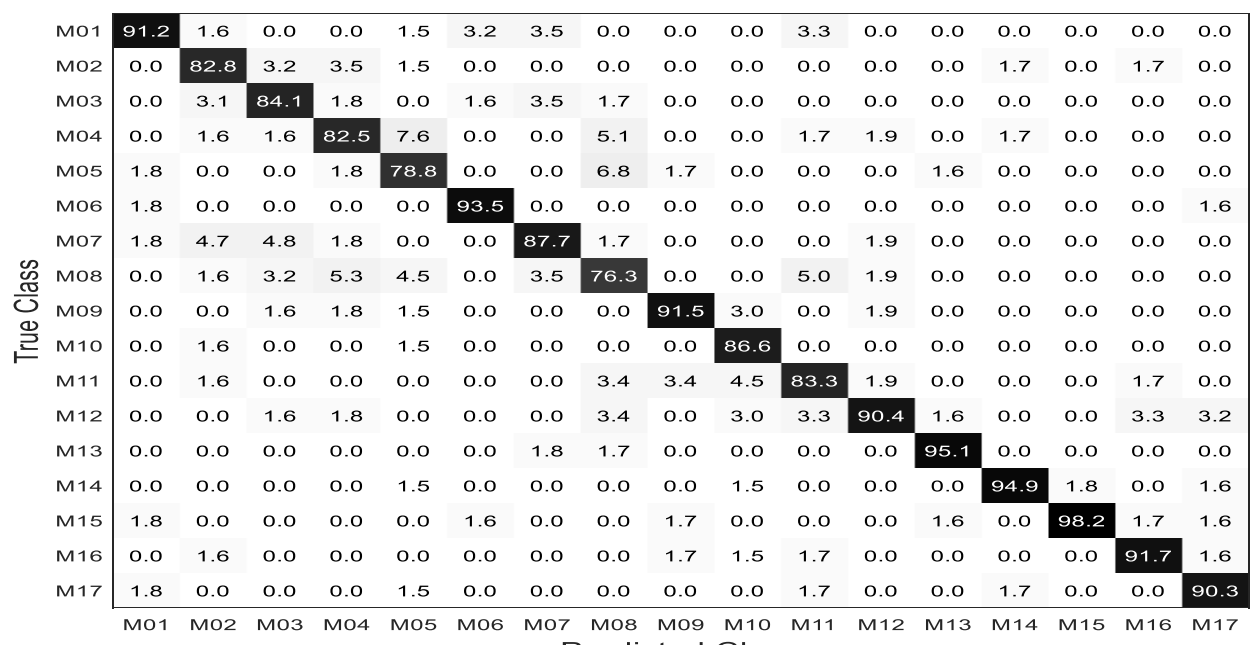

Predicted Class

Figure 4. Confusion matrix of CNN model 2 across 10 healthy subjects (\%)

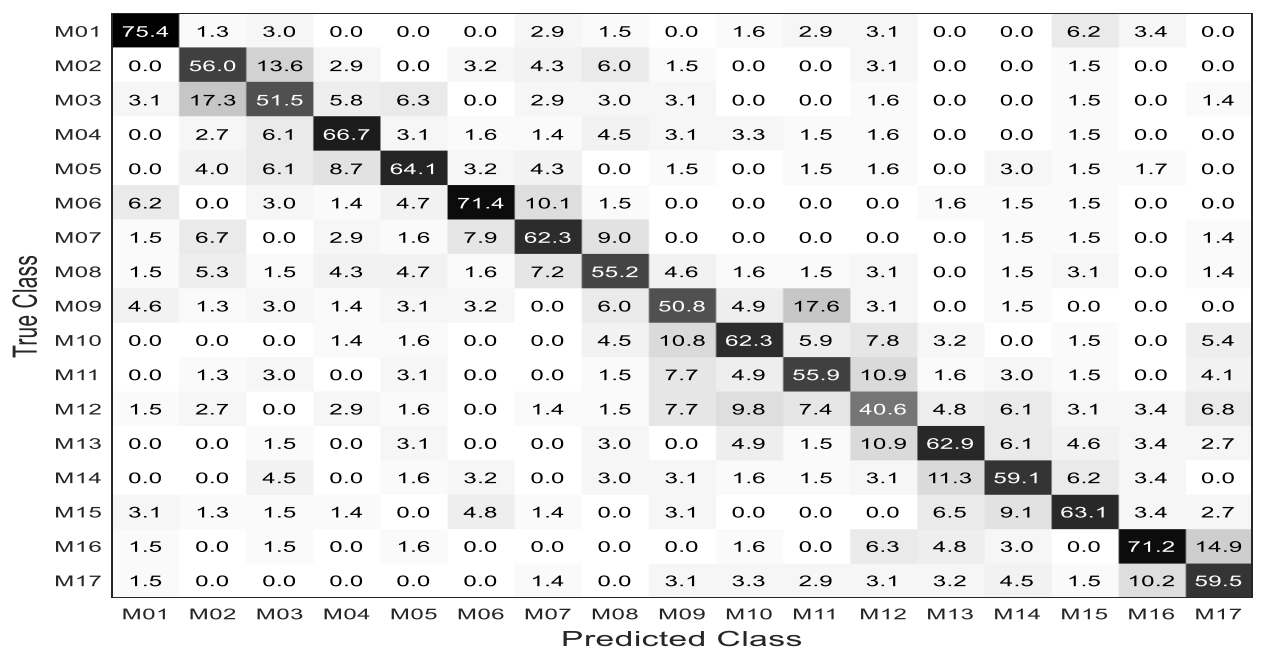

Figure 5. Confusion matrix of CNN model 2 across 11 amputee subjects (\%)

\section{CONCLUSION}

In current work, the performance of featureless EMG pattern recognition in classifying the 17 hand and wrist movements are presented. Two CNN architectures have been proposed for performance evaluation. In this framework, CNN took the spectrogram images directly for classification without the need of feature extraction. The performances of proposed CNN are evaluated using the EMG data of 11 amputees and 10 healthy subjects. Our results showed that the CNN model 2 offered the highest mean classification accuracy of $88.04 \%$ for recognizing the 17 hand and wrist movements. It indicates that the additional convolutional layer can improve the classification results, but with extra computational cost. However, it is worth noting that the best classification accuracy is below 90\%, which needs to be improved for real time application. In future, a CNN architecture should be designed deeper in order to ensure better classification performance. 


\section{ACKNOWLEDGEMENTS}

The authors would like to thank the Universiti Teknikal Malaysia Melaka (UTeM), Skim Zamalah UTeM and Minister of Higher Education Malaysia (MOHE) for funding research under grant FRGS/1/2017/TK04/FKE-CeRIA/F00334.

\section{REFERENCES}

[1] Yousefi J, Hamilton-Wright A. Characterizing EMG data using machine-learning tools. Computers in biology and medicine. 2014; 51: 1-13.

[2] Zhang X, Zhou P. High-density myoelectric pattern recognition toward improved stroke rehabilitation. IEEE Transactions on Biomedical Engineering. 2012; 59(6): 1649-1657.

[3] Chen X, Zhu X, Zhang D. A discriminant bispectrum feature for surface electromyogram signal classification. Medical Engineering and Physics. 2010; 32(2): 126-135.

[4] Purushothaman G, Ray KK. EMG based man-machine interaction-A pattern recognition research platform. Robotics and Autonomous Systems. 2014; 62(6): 864-870.

[5] Atzori M, Gijsberts A, Castellini C, Caputo B, Hager AG, Elsig S, Giatsidis G, Bassetto F, Müller H. Electromyography data for non-invasive naturally-controlled robotic hand prostheses. Scientific data. 2014; 1: 140053.

[6] Al Omari F, Hui J, Mei C, Liu G. Pattern recognition of eight hand motions using feature extraction of forearm EMG signal. Proceedings of the National Academy of Sciences, India Section A: Physical Sciences. 2014; 84(3): 473-480.

[7] Phinyomark A, Nuidod A, Phukpattaranont P, Limsakul C. Feature extraction and reduction of wavelet transform coefficients for EMG pattern classification. Elektronika ir Elektrotechnika. 2012; 122(6): 27-32.

[8] Phinyomark A, Phukpattaranont P, Limsakul C. Feature reduction and selection for EMG signal classification. Expert Systems with Applications. 2012; 39(8): 7420-7431.

[9] Too J, Abdullah AR, Saad NM, Ali NM, and Tee W. A New Competitive Binary Grey Wolf Optimizer to Solve the Feature Selection Problem in EMG Signals Classification. Computers. 2018; 7(4): 58.

[10] Gonzalez-Izal M, Malanda A, Navarro-Amezqueta I, Gorostiaga EM, Mallor F, Ibanez J, Izquierdo M. EMG spectral indices and muscle power fatigue during dynamic contractions. Journal of Electromyography and Kinesiology. 2010; 20(2): 233-240.

[11] Rafiee J, Rafiee MA, Yavari F, Schoen MP. Feature extraction of forearm EMG signals for prosthetics. Expert Systems with Applications. 201; 38(4): 4058-4067.

[12] Wang J, Zhuang J, Duan L, Cheng W. A multi-scale convolution neural network for featureless fault diagnosis. InFlexible Automation (ISFA), International Symposium on 2016 Aug 1 (pp. 65-70). IEEE.

[13] Too J, Abdullah AR, Saad NM, Ali NM. Feature Selection Based on Binary Tree Growth Algorithm for the Classification of Myoelectric Signals. Machines. 2018; 6(4): 65.

[14] Längkvist M, Karlsson L, Loutfi A. A review of unsupervised feature learning and deep learning for time-series modeling. Pattern Recognition Letters. 2014; 42: 11-24.

[15] Ashwin JS, Manoharan N. Convolutional Neural Network Based Target Recognition for Marine Search. Indonesian Journal of Electrical Engineering and Computer Science. 2017; 8(2): 561-563.

[16] Verstraete D, Ferrada A, Droguett EL, Meruane V, Modarres M. Deep Learning Enabled Fault Diagnosis Using Time-Frequency Image Analysis of Rolling Element Bearings. Shock and Vibration. 2017;2017.

[17] AbdelMaseeh M, Chen TW, Stashuk DW. Extraction and classification of multichannel electromyographic activation trajectories for hand movement recognition. IEEE Transactions on Neural Systems and Rehabilitation Engineering. 2016; 24(6): 662-673.

[18] Atzori M, Cognolato M, Müller H. Deep learning with convolutional neural networks applied to electromyography data: a resource for the classification of movements for prosthetic hands. Frontiers in neurorobotics. 2016 ; 10 : 9.

[19] Pizzolato S, Tagliapietra L, Cognolato M, Reggiani M, Müller H, Atzori M. Comparison of six electromyography acquisition setups on hand movement classification tasks. PloS one. 2017; 12(10): e0186132.

[20] Tsai AC, Luh JJ, Lin TT. A novel STFT-ranking feature of multi-channel EMG for motion pattern recognition. Expert Systems with Applications. 2015; 42(7): 3327-3341.

[21] Sengur A, Akbulut Y, Guo Y, Bajaj V. Classification of amyotrophic lateral sclerosis disease based on convolutional neural network and reinforcement sample learning algorithm. Health information science and systems. 2017; 5(1): 9.

[22] Nawawi MA, Ismail FS, Selamat H. Comprehensive Pineapple Segmentation Techniques with Intelligent Convolutional Neural Network. Indonesian Journal of Electrical Engineering and Computer Science. 2018; 10(3): 1098-1195.

[23] Schmidhuber J. Deep learning in neural networks: An overview. Neural networks. 2015; 61: 85-117.

[24] Sengur A, Gedikpinar M, Akbulut Y, Deniz E, Bajaj V, Guo Y. DeepEMGNet: an application for efficient discrimination of ALS and normal EMG signals. InInternational Conference Mechatronics 2017; 619-625.

[25] Jamil N, Almisreb AA, Ariffin SM, Din NM, Hamzah R. Can Convolution Neural Network (CNN) Triumph in Ear Recognition of Uniform Illumination Invariant. Indonesian Journal of Electrical Engineering and Computer Science. 2018; 11(2). 